\title{
One model of modified gravity with dynamical torsion and its cosmological consequences
}

\author{
Vasilisa Nikiforova ${ }^{1, \star}$ \\ ${ }^{1}$ Institute for Nuclear Research of the Russian Academy of Sciences, 60th October Anniversary Prospect \\ 7a, 117312 Moscow, Russia
}

\begin{abstract}
We consider a model belonging to the class of Poincarè gauge gravities. The model is free of ghosts, tachyons and gradient instabilities about Minkowski and torsionless Einstein backgrounds of sufficiently small curvature. At zero cosmological constant, the model admits a self-accelerating solution with non-Riemannian connection. We study scalar perturbations about the self-accelerating solution and find that the number of scalar modes is the same as in Minkowski background; moreover, in the limit of small effective cosmological constant and below the UV cutoff of the low energy effective theory, the scalar sector does not have pathologies like ghosts or rapid gradient instabilities.
\end{abstract}

\section{Introduction}

Modified gravity recently has become of considerable interest mostly in the cosmological context as a viable alternative to General Relativity on cosmological scales. The idea is to replace General Relativity with some other theory which could drive the cosmological acceleration without dark energy.

There are many approaches to modify gravity in IR, see a comprehensive review [1]. These include theories with extra dimensions [2-6] (for a review see Ref. [7]), $f(R)$ and scalar-tensor gravities (for reviews see Refs. [8] and [9]), theories with massive gravitons [10-13] (for a review see Ref. [14]) and theories with explicitly broken Lorentz invariance [15-19] (for a review see Ref. [20]). However, self-accelerating solutions in IR-modified gravities are often plagued with instabilities. In particular, the Minkowski background may be stable, but extra modes may appear in curved backgrounds. They usually have wrong sign kinetic terms and are known as Boulware-Deser modes [21]. Even if the Boulware-Deser modes do not appear, the spectrum can have ghosts or gradient instabilities. One famous example is the DGP model [2], which admits both Minkowski and self-accelerating backgrounds $[22,23]$. The former one has only healthy degrees of freedom in the spectrum, while the self-accelerating background has ghost instability [24-28].

Yet another class of theories which are healthy on the Minkowski background [29-32] and may serve as candidates for the consistent infrared modification of gravity are Poincarè gauge gravities. These theories treat the vierbein and connection as independent variables, and the Lagrangians include bilinear terms in the full curvature tensor, as well as bilinear terms in the torsion. The spectrum of these theories about the Minkowski background contains both massless degrees of freedom corresponding to the standard graviton and massive degrees of freedom related to the propagating torsion

^e-mail: nikiforovavas@mail.ru 
[31]; the gravitational interaction in these theories is described by both Newtonian and Yukawa terms [30]. The model which we consider in this paper belongs to the class of Poincarè gauge gravities.

This model was studied earlier in Refs. [33-36]. It was shown that ghosts, gradient instabilities and tachyons are absent in the spectrum about the Minkowski background, in de Sitter and anti-de Sitter spaces and in arbitrary torsionless Einstein backgrounds of sufficiently small curvature, provided that certain constraints on the couplings are satisfied [31,33,34]. This model admits the self-accelerating solution [36]. It is certainly of interest to understand the properties of perturbations about this solution. Our purpose is to study whether this theory exhibits the Boulware-Deser phenomenon and/or other instabilities in self-accelerating background. The known examples show that possible problems occur in the scalar sector of perturbations. Therefore, we focus in this paper on the scalar sector.

This paper is organized as follows. In Section 2 we present the Lagrangian, write the field equations and derive the generalized Bianchi identities. In Section 3 we present the self-accelerating solution with effective cosmological constant which can be made small by an appropriate choice of parameters. In Section 4 we study perturbations about this solution. We find that there are no extra modes as compared to the Minkowski background. We find also that in the limit of small effective cosmological constant and within the range of validity of the effective low energy theory there are actually no propagating degrees of freedom at all.

\section{The Model}

\subsection{Action and field equations}

We make use of the tetrad formalism and consider vierbein $\left(e_{\mu}^{i}\right)$ and connection $\left(A_{i j \mu}=-A_{j i \mu}\right)$ as independent fields ${ }^{1} ; \mu=(0,1,2,3)$ is the space-time index, and $i, j=(0,1,2,3)$ are the tangent space indices. In tangent space basis the indices are raised and lowered using the Minkowski metric $\eta_{i j}$, so we do not distinguish upper and lower tangent space indices in what follows, if this does not lead to an ambiguity. The signature of metric is $(-,+,+,+)$.

The action of the model is

$$
L=\frac{3}{2}(\tilde{\alpha} F-\alpha R)+c_{3} F^{i j} F_{i j}+c_{4} F^{i j} F_{j i}+c_{5} F^{2}+c_{6}(\epsilon \cdot F)^{2},
$$

where $\alpha, \tilde{\alpha}, c_{3}, c_{4}, c_{5}, c_{6}$ are the coupling constants, $e \equiv \operatorname{det}\left(e_{\mu}^{i}\right) ; F_{i j k l}$ is the curvature tensor constructed with the connection $A_{i j \mu}$,

$$
\begin{gathered}
F_{i j k l}=e_{k}^{\mu} e_{l}^{v}\left(\partial_{\mu} A_{i j v}-\partial_{v} A_{i j \mu}+A_{i m \mu} A_{m j v}-A_{j m \mu} A_{m i v}\right) \\
F_{i j}=\eta^{k l} F_{i k j l}, \quad F=\eta^{i j} F_{i j}, \quad \epsilon \cdot F \equiv \epsilon^{i j k l} F_{i j k l}
\end{gathered}
$$

$\epsilon_{i j k l}$ is the Levi-Civita symbol defined in such a way that $\epsilon^{0123}=-\epsilon_{0123}=1 ; R_{i j k l}$ is the Riemann curvature tensor,

$$
\begin{gathered}
R_{i j k l}=e_{k}^{\mu} e_{l}^{v}\left(\partial_{\mu} \omega_{i j v}-\partial_{\nu} \omega_{i j \mu}+\omega_{i m \mu} \omega_{m j v}-\omega_{j m \mu} \omega_{m i v}\right) ; \\
R_{i j}=\eta^{k l} R_{i k j l}, \quad R=\eta^{i j} R_{i j},
\end{gathered}
$$

where $\omega_{i j \mu}$ is the Riemannian spin-connection. The latter is expressed in terms of the vierbein as follows:

$$
\omega_{i j \mu} \equiv \omega_{i j k} e_{\mu}^{k}=\frac{1}{2}\left(C_{i j k}-C_{j i k}-C_{k i j}\right) e_{\mu}^{k},
$$

\footnotetext{
${ }^{1}$ The formalism used in this section is largely the one developed in Ref. [35].
} 
where

$$
C_{i j k}=e_{j}^{\mu} e_{k}^{v}\left(\partial_{\mu} e_{i v}-\partial_{\nu} e_{i \mu}\right) .
$$

The connection $A_{i j \mu}$ can be represented as a sum

$$
A_{i j \mu}=\omega_{i j \mu}+K_{i j \mu},
$$

where $K_{i j \mu}$ is the contorsion tensor.

It was shown that there are no pathological degrees of freedom in the Minkowski background [31] if one imposes the following conditions on the couplings,

$$
\begin{aligned}
& c_{3}+c_{4}=-3 c_{5}, \\
& \alpha<0, \quad \tilde{\alpha}>0, \quad c_{5}<0, \quad c_{6}>0 .
\end{aligned}
$$

We consider this case in that follows. In Ref. [34] it was found that there are three propagating modes at the linear level in the Minkowski background: the massless spin-2 mode, the massive spin-2 mode with mass

$$
m^{2}=\frac{\tilde{\alpha}(\tilde{\alpha}-\alpha)}{2 \alpha c_{5}}
$$

and the massive spin-0 mode with mass

$$
m_{0}^{2}=\frac{\tilde{\alpha}}{16 c_{6}} .
$$

It is worth pointing out that there are two helicity- 0 modes, the scalar of mass (5) and longitudinal part of massive spin-2 mode. From the point of view of (3+1)-decomposition of Sec. 4.1, these are scalar perturbations. There are no ghosts or tachyons in the Minkowski background. It was also shown that in the theory equipped with the cosmological constant, the perturbations are healthy in maximally symmetric backgrounds, and in torsionless Einstein backgrounds of sufficiently small curvature as well.

There are two sets of field equations in our model. One consists of the gravitational field equations obtained by varying the action with respect to vierbein,

$$
\begin{aligned}
\mathcal{G}_{i j} \equiv & \frac{3}{2} \tilde{\alpha}\left(F_{i j}-\frac{1}{2} \eta_{i j} F\right)-\frac{3}{2} \alpha\left(R_{i j}-\frac{1}{2} \eta_{i j} R\right)+c_{3}\left(F_{k i} F_{k j}+F_{k l} F_{k i l j}\right) \\
& +c_{4}\left(F_{i k} F_{k j}+F_{l k} F_{k i l j}\right)+2 c_{5} F F_{i j}+2 c_{6} \epsilon_{k l m i} F_{k l m j}(\epsilon \cdot F)-\frac{1}{2} \eta_{i j} L^{(2)}=0,
\end{aligned}
$$

where

$$
L^{(2)}=c_{3} F_{i j} F_{i j}+c_{4} F_{i j} F_{j i}+c_{5} F^{2}+c_{6}(\epsilon \cdot F)^{2}
$$

is the part in the Lagrangian which is bilinear in $F_{i j k l}$. Another set of equations is obtained by varying the action with respect to the connection $A_{i j \mu}$,

$$
\begin{aligned}
\mathcal{T}_{i j k} \equiv & H_{i j k}+\left\{\left[\eta_{i k}\left(D_{m} P_{j m}-\frac{2}{3} D_{j} P\right)-D_{i} P_{j k}\right]-\left[\eta_{j k}\left(D_{m} P_{i m}-\frac{2}{3} D_{i} P\right)-D_{j} P_{i k}\right]\right\} \\
& +4 c_{6} \epsilon_{i j k m} D_{m}(\epsilon \cdot F)+S_{i j k}=0,
\end{aligned}
$$

where $P_{i j}$ and $P$ are defined as follows,

$$
\begin{aligned}
& P_{i j}=c_{3} F_{i j}+c_{4} F_{j i}, \\
& P=\eta^{i j} P_{i j}
\end{aligned}
$$


the covariant derivative $D_{i}$ involves the connection $A_{i j \mu}$,

$$
D_{i} B_{j} \equiv e_{i}^{\mu} D_{\mu} B_{j}=e_{i}^{\mu}\left(\partial_{\mu} B_{j}-A_{l j \mu} B_{l}\right)
$$

$S_{i j k}$ is defined as

$$
S_{i j k}=\frac{2}{3 \tilde{\alpha}} H_{m n k}\left(\eta_{i m} P_{j n}-\eta_{j m} P_{i n}-\frac{2}{3} \eta_{i m} \eta_{j n} P+2 c_{6} \epsilon_{i j m n}(\epsilon \cdot F)\right)
$$

and $H_{i j k}$ is written in terms of contorsion as follows,

$$
\frac{2}{3 \tilde{\alpha}} H_{i j k}=K_{i k j}-K_{j k i}-K_{i l l} \eta_{j k}+K_{j l l} \eta_{i k} .
$$

Because of the invariance of the action under space-time gauge transformations and local Lorentz transformations, not all of field equations are independent.

\subsection{Generalized Bianchi identities}

The local Lorentz and space-time gauge invariance can be used to find the identities relating the field equations (6), (7) with each other. Let us start with the space-time gauge invariance. Making an infinitesimal gauge transformation, $x^{\prime \mu}=x^{\mu}+\xi^{\mu}$, we find the variations of $e_{\mu}^{i}$ and $A_{i j \mu}$ :

$$
\begin{aligned}
& \delta e_{\mu}^{i}=-\xi^{\lambda} \partial_{\lambda} e_{\mu}^{i}-e_{\lambda}^{i} \partial_{\mu} \xi^{\lambda}=-\xi^{\lambda} \nabla_{\lambda} e_{\mu}^{i}-e_{\lambda}^{i} \nabla_{\mu} \xi^{\lambda}, \\
& \delta A_{i j \mu}=-A_{i j \lambda} \nabla_{\mu} \xi^{\lambda}-\xi^{\lambda} \nabla_{\lambda} A_{i j \mu},
\end{aligned}
$$

where $\nabla_{\mu}$ is the covariant derivative involving the Christoffel symbols,

$$
\nabla_{\mu} e_{v}^{i}=\partial_{\mu} e_{v}^{i}-\Gamma_{\mu \nu}^{\lambda} e_{\lambda}^{i}
$$

Evaluating the variation of the action with respect to $e_{\mu}^{i}$ and $A_{i j \mu}$ of the form (8), (9) we find, upon integrating by parts, the following identity:

$$
-2 \mathcal{G}_{i k} e_{k v} \nabla_{\mu} e^{i v}-2 \nabla_{v}\left(\mathcal{G}_{i k} e_{k \mu} e^{i v}\right)-e^{k v} \nabla_{\mu} A_{i j v} \mathcal{T}_{i j k}+\nabla_{v}\left(e^{k v} A_{i j \mu} \mathcal{T}_{i j k}\right)=0
$$

(see eqs. (6) and (7) for definitions of $\mathcal{G}_{i j}$ and $\mathcal{T}_{i j k}$ ).

Another identity comes from the local Lorentz invariance. The model discussed is a gauge theory with Lorentz group as a gauge group. The variations of $e_{\mu}^{i}$ and $A_{i j \mu}$ under the infinitesimal Lorentz transformation are:

$$
\begin{aligned}
& \delta e_{i}^{\mu}=\omega_{i j} e_{j}^{\mu}, \\
& \delta A_{i j \mu}=-\partial_{\mu} \omega_{i j}+\omega_{i p} A_{p j \mu}-\omega_{j p} A_{p i \mu},
\end{aligned}
$$

where $\omega_{i j}=-\omega_{j i}$ are the parameters of transformation. The invariance of the action under this transformation gives,

$$
\left(\mathcal{G}_{i j}-\mathcal{G}_{j i}\right)+\nabla_{v}\left(\mathcal{T}_{i j k} e^{k v}\right)+2 e^{k v}\left(\mathcal{T}_{i p k} A_{j p v}-\mathcal{T}_{j p k} A_{i p v}\right)=0
$$

The left hand side of eq. (12) contains two free indices, $i$ and $j$, and is antisymmetric in them. 


\section{The self-accelerating cosmological solution}

The model (1) admits a self-accelerating solution [36],

$$
e_{0}^{\tilde{0}}=1, \quad e_{b}^{\tilde{a}}=e^{\lambda t} \delta_{b}^{\tilde{a}}, \quad A_{\tilde{0} \tilde{a} \tilde{b}}=f \delta_{\tilde{a} \tilde{b}}, \quad A_{\tilde{a} \tilde{b} \tilde{c}}=g \varepsilon_{\tilde{a} \tilde{b} \tilde{c}},
$$

with constant $\lambda, f$ and $g$, where $a, \tilde{a}=(1,2,3)$, tilde denotes tangent space indices, and space-time indices do not have tilde. The parameters $f, g$ and $\lambda$ are related to couplings as follows,

$$
\begin{aligned}
\tilde{\alpha}\left(f^{2}-g^{2}\right)-\alpha \lambda^{2}-16 c_{6} g^{2} \lambda^{2}+64 c_{6} f^{2} g^{2} & =0, \\
\tilde{\alpha}(f+\lambda)-4 c_{5} g^{2} \lambda-32 c_{6} g^{2}(\lambda-2 f) & =0, \\
\tilde{\alpha}\left(f^{2}-g^{2}-\lambda f\right)-2 \alpha \lambda^{2} & =0 .
\end{aligned}
$$

One finds that if the following condition is satisfied,

$$
c_{5}+16 c_{6}<0,
$$

then there exists the self-accelerating solution with $\lambda>0, f<0$. The sign of $g$ can be arbitrary, since $g$ is P-odd.

The small value of the effective cosmological constant $\lambda$ is obtained provided that there is a hierarchy between the dimensionless couplings: for $c_{6}=O(\lambda), c_{5}=O\left(\lambda^{-1}\right)$ the parameter $\lambda$ is the smallest parameter of the problem [36]. It is straightforward to see from eqs. (14a) - (14c) that in this case the parameters $f, g$ and $\lambda$ are related to couplings as follows:

$$
\begin{aligned}
& \lambda=\left(-\frac{c_{6} \tilde{\alpha}^{2}}{c_{5}^{3}}\right)^{1 / 4}, \\
& f=-\frac{\tilde{\alpha}^{1 / 2}}{4\left(-c_{5} c_{6}\right)^{1 / 4}}, \\
& g= \pm f+O(\lambda) .
\end{aligned}
$$

The effective cosmological constant can also be written in terms of the masses (4), (5) of excitations about the Minkowski background:

$$
\lambda=m\left(\frac{m}{m_{0}}\right)^{1 / 2} \frac{(-\alpha)^{3 / 4}}{2^{1 / 4}(\tilde{\alpha}-\alpha)^{3 / 4}} .
$$

We point out that the solution (13) does not require any explicit cosmological constant in the Lagrangian (1); the self-acceleration is generated by torsion.

\section{Perturbations}

Our purpose is to study small perturbations about the self-accelerating background (13). To this end, we work with conformal time

$$
\eta=\int e^{-\lambda t} d t=-\frac{1}{\lambda} e^{-\lambda t}
$$

Let us also change the notation and denote the vierbein of the original theory by $\hat{e}_{\mu}^{i}$. It is convenient to make the following conformal transformation,

$$
\hat{e}_{\mu}^{i}=\mathrm{e}^{\phi} e_{\mu}^{i}, \quad \hat{e}_{j}^{v}=\mathrm{e}^{-\phi} e_{j}^{v},
$$


where

$$
\mathrm{e}^{\phi}=a(\eta)=-\frac{1}{\lambda \eta}
$$

Note that

$$
\phi^{\prime}=\lambda \mathrm{e}^{\phi},
$$

where prime denotes derivative with respect to conformal time. We do not make any scaling of connection, and keep the notation $A_{i j \mu}$ unchanged. Upon the conformal transformation, the background has flat metric and non-trivial connection,

$$
e_{\mu}^{i}=\delta_{\mu}^{i}, \quad A_{\tilde{a} \tilde{a} \tilde{b}}=f \delta_{\tilde{a} \tilde{b}}, \quad A_{\tilde{a} \tilde{b} \tilde{c}}=g \varepsilon_{\tilde{a} \tilde{b} \tilde{c}}
$$

Equation (6) then reads

$$
\begin{aligned}
& \frac{3}{2} \tilde{\alpha} \mathrm{e}^{-2 \phi}\left(F_{i j}-\frac{1}{2} \eta_{i j} F\right)-\frac{3}{2} \alpha \mathrm{e}^{-2 \phi}\left(R_{i j}-\frac{1}{2} \eta_{i j} R\right) \\
& -\frac{3}{2} \alpha \mathrm{e}^{-2 \phi}\left[-2 e_{i}^{\mu} e_{j}^{\nu} \nabla_{\mu} \nabla_{\nu} \phi-\eta_{i j} g^{\mu \nu} \nabla_{\mu} \nabla_{\nu} \phi+2 e_{i}^{\mu} e_{j}^{\nu} \partial_{\mu} \phi \partial_{\nu} \phi-2 \eta_{i j} g^{\mu \nu} \partial_{\mu} \phi \partial_{\nu} \phi\right. \\
& \left.-\eta_{i j}\left(-3 g^{\mu \nu} \nabla_{\mu} \nabla_{\nu} \phi-3 g^{\mu \nu} \partial_{\mu} \phi \partial_{\nu} \phi\right)\right] \\
& +\mathrm{e}^{-4 \phi} c_{3}\left(F_{k i} F_{k j}+F_{k l} F_{k i l j}\right)+\mathrm{e}^{-4 \phi} c_{4}\left(F_{i k} F_{k j}+F_{l k} F_{k i l j}\right)+2 \mathrm{e}^{-4 \phi} c_{5} F F_{i j} \\
& +2 \mathrm{e}^{-4 \phi} c_{6} \epsilon_{k l m i} F_{k l m j}(\epsilon \cdot F)-\mathrm{e}^{-4 \phi} \frac{1}{2} \eta_{i j} L^{(2)}=0,
\end{aligned}
$$

while eq. (7) gives

$$
\begin{aligned}
& \mathrm{e}^{-\phi}\left(H_{i j k}+3 \tilde{\alpha} \eta_{i k} e_{j}^{\mu} \partial_{\mu} \phi-3 \tilde{\alpha} \eta_{j k} e_{i}^{\mu} \partial_{\mu} \phi\right) \\
& +\mathrm{e}^{-3 \phi}\left\{\left[\eta_{i k}\left(D_{m} P_{j m}-\frac{2}{3} D_{j} P\right)-D_{i} P_{j k}\right]-\left[\eta_{j k}\left(D_{m} P_{i m}-\frac{2}{3} D_{i} P\right)-D_{j} P_{i k}\right]\right\} \\
& \quad+4 \mathrm{e}^{-3 \phi} c_{6} \epsilon_{i j k m} D_{m}(\epsilon \cdot F)+\mathrm{e}^{-3 \phi} S_{i j k}=0 .
\end{aligned}
$$

\section{$4.1(3+1)$-decomposition}

After conformal transformation for the vierbein (15) we have

$$
e_{\mu}^{i}=\delta_{\mu}^{i}+\epsilon_{\mu}^{i}
$$

where $\epsilon_{\mu}^{i}$ is the first order quantity. As mentioned above, the model is invariant under local Lorentz and space-time gauge transformations. The former can be used to make $\epsilon_{i \mu}$ symmetric; this, in particular, means that

$$
e_{j}^{\mu}=\delta_{j}^{\mu}-\epsilon_{i v} \delta_{j}^{v} \delta^{i \mu}
$$

while the space-time gauge invariance can be used to choose the gauge $e_{\tilde{0} a}=0$ and the conformal Newtonian gauge.

The full contorsion tensor is written as follows,

$$
K_{i j k}=K_{i j k(0)}+k_{i j k}
$$

where $K_{i j k(0)}=e_{k}^{\mu} K_{i j \mu(0)}=e_{k}^{\mu}\left(A_{i j \mu(0)}-\omega_{i j \mu(0)}\right)=e_{k}^{\mu} A_{i j \mu(0)}$ is the background quantity constructed from (16) and $k_{i j k}$ is the first order perturbation. 
It is convenient to make the 3-dimensional Fourier decomposition. We use the same notation for the Fourier-transformed variables and replace spatial derivatives $\partial_{\tilde{a}} \equiv e_{\tilde{a}}^{\mu} \partial_{\mu}$ with $i k_{\tilde{a}}$, where $\mathbf{k}$ is the 3-dimensional momentum. Since the background (16) is invariant under spatial rotations, it is convenient to use $(3+1)$-decomposition of perturbations. This means that we decompose any 3dimensional tensor into its irreducible components with respect to the small group $S O(2)$ of rotations around the spatial momentum. These irreducible components form sectors with particular values of helicity: scalar sector (helicity-0), vector sector (helicity-1) and tensor sector (helicity-2). Since the field equations are linear, these sectors can be considered separately.

Tensor $k_{i j k}=-k_{j i k}$ contains helicity $0,1,2$ components only. The helicity-2 components form the tensor sector:

$$
\begin{aligned}
& k_{\tilde{a} \tilde{a} \tilde{b}}=-k_{\tilde{a} \tilde{0} \tilde{b}}=\tau_{\tilde{a} \tilde{b} \tilde{b}}, \\
& k_{\tilde{a} \tilde{b} \tilde{c}}=k_{\tilde{a}} N_{\tilde{b} \tilde{c}}-k_{\tilde{b}} N_{\tilde{a} \tilde{c}},
\end{aligned}
$$

where $\tau_{\tilde{a} \tilde{b}}$ and $N_{\tilde{a} \tilde{b}}$ are symmetric, transverse and traceless. All other components of $k_{i j k}$ vanish in the tensor sector.

The non-vanishing helicity-1 components of contorsion are

$$
\begin{aligned}
& k_{\tilde{0} \tilde{a} \tilde{0}}=-k_{\tilde{a} \tilde{0} \tilde{0}}=\zeta_{\tilde{a}}, \\
& k_{\tilde{0} \tilde{a} \tilde{b}}=-k_{\tilde{a} \tilde{0} \tilde{b}}=k_{\tilde{a}} v_{\tilde{b}}+k_{\tilde{b}} \mu_{\tilde{a}}, \\
& k_{\tilde{a} \tilde{b} \tilde{0}}=k_{\tilde{a}} K_{\tilde{b}}-k_{\tilde{b}} K_{\tilde{a}}, \\
& k_{\tilde{a} \tilde{b} \tilde{c}}=k_{\tilde{a}} k_{\tilde{c}} \alpha_{\tilde{b}}-k_{\tilde{b}} k_{\tilde{c}} \alpha_{\tilde{a}}+\eta_{\tilde{a} \tilde{c}} L_{\tilde{b}}-\eta_{\tilde{b} \tilde{c}} L_{\tilde{a}},
\end{aligned}
$$

all 3-vectors here are transverse. The helicity-0 components form the scalar sector:

$$
\begin{aligned}
& k_{\tilde{0} \tilde{a} \tilde{0}}=-k_{\tilde{a} \tilde{0} \tilde{0}}=k_{\tilde{a}} \xi, \\
& k_{\tilde{0} \tilde{a} \tilde{b}}=-k_{\tilde{a} \tilde{0} \tilde{b}}=k_{\tilde{a}} k_{\tilde{b}} \chi+\delta_{\tilde{a} \tilde{b}} \sigma+\epsilon_{\tilde{a} \tilde{b} \tilde{c}} k_{\tilde{c}} \rho, \\
& k_{\tilde{a} \tilde{b} \tilde{0}}=\epsilon_{\tilde{a} \tilde{b} \tilde{c}} k_{\tilde{c}} \theta, \\
& k_{\tilde{a} \tilde{b} \tilde{c}}=\epsilon_{\tilde{a} \tilde{b} \tilde{d}} k_{\tilde{c}} k_{\tilde{d}} Q+\left(k_{\tilde{a}} \epsilon_{\tilde{b} \tilde{c} \tilde{d}}-k_{\tilde{b}} \epsilon_{\tilde{a} \tilde{c} \tilde{d}}\right) k_{\tilde{d}} u+\left(k_{\tilde{a}} \delta_{\tilde{b} \tilde{c}}-k_{\tilde{b}} \delta_{\tilde{a} \tilde{c}}\right) M .
\end{aligned}
$$

There are altogether 24 components of tensor $k_{i j k}$. They break up into the 4 tensor components (two components from each of $\tau_{\tilde{a} \tilde{b}}$ and $N_{\tilde{a} \tilde{b}}$ ), 12 components in vector sector (two components from each of six transverse vectors $\zeta_{\tilde{a}}, v_{\tilde{a}}, \mu_{\tilde{a}}, \kappa_{\tilde{a}}, \alpha_{\tilde{a}}$ and $\left.L_{\tilde{a}}\right)$ and 8 scalar components $(\xi, \chi, \sigma, \rho, \theta, Q, u$ and $M)$.

In what follows the space-time indices in the first order quantities are raised and lowered with Minkowski metric. The vierbein perturbations (in conformal Newtonian gauge with $\epsilon_{\mu \nu}=\epsilon_{v \mu}$ and $\left.e_{\tilde{0} a}=0\right)$ are:

$$
\begin{aligned}
& \epsilon_{\tilde{0} \tilde{0}}=-\Phi, \\
& \epsilon_{\tilde{0} \tilde{a}}=0, \\
& \epsilon_{\tilde{a} \tilde{b}}=\Psi \delta_{\tilde{a} \tilde{b}}+i\left(k_{\tilde{a}} W_{\tilde{b}}+k_{\tilde{b}} W_{\tilde{a}}\right)+\pi_{\tilde{a} \tilde{b}},
\end{aligned}
$$

where $W_{\tilde{a}}$ is a transverse vector (vector sector, 2 components), $\pi_{\tilde{a} \tilde{b}}$ is a transverse traceless tensor (tensor sector, 2 components), while $\Phi$ and $\Psi$ belong to the scalar sector ( 2 components).

\subsection{Scalar sector}

In this paper we study the scalar sector of perturbations. In this sector we have in total 14 scalar field equations; 6 of them are second order in time derivatives and 8 are first order. Not all of these 14 
equations are independent, as there are Bianchi identities (10), (12). The left hand side of eq. (10) contains one free index $\mu$ and thus can be decomposed into two scalar identities: with $\mu=0$ and $\mu=a$. Furthermore, the left hand side of eq. (12) is decomposed into two scalar identities, one for $i=a, j=0$ and another for $i=a, j=b$. In total we have 4 scalar Bianchi identities relating 14 field equations with each other. Using these identities we can exclude 4 second order equations from consideration. Thus, we have 10 equations. Off hand, two of these equations are second order. However, a sum of each of these second order equations with a linear combination of time-derivatives of the 8 first order equations is a first order equation. Therefore, at this stage we have 10 first order equations for 10 variables characterizing scalar perturbations. This is not the whole story, though.

\subsubsection{Number of propagating modes}

Let us count the number of propagating degrees of freedom. The variables in the resulting system of 10 first order equations are of two types: the variables of first group,

$$
\sigma, \Phi, \xi, \theta
$$

enter the linearized equations without time derivatives. The variables of the second group,

$$
\Psi, \chi, \rho, Q, u, M,
$$

enter with first time derivatives. Then four of the ten equations can be used to express non-dynamical variables $\sigma, \Phi, \xi$ and $\theta$ through the variables (19). After that, there remain 6 first order equations for the 6 variables (19).

Thus, naively we have 3 degrees of freedom. But this is not the case: two linear combinations of the 6 first order equations actually do not contain time derivatives, i.e., they are algebraic equations. These two equations are used to express two of the variables (19) in terms of the other 4 variables (say, $\Psi, \chi, Q, u$ ). There remain 4 first order equations for these 4 variables. Thus, we have 2 degrees of freedom, the same number as in the Minkowski background. We concude that the self-accelerating background does not exhibit the Boulevare-Deser phenomenon.

\subsubsection{Limit of small $\lambda$}

Let us now consider the small- $\lambda$ limit (see Sec. 3). We seek for solutions in the form $F \sim e^{i \int \omega d \eta}$, where $F$ is any of the variables $\Psi, \chi, Q, u$, and

$$
\omega \gg \lambda, \quad k^{2} \gg \lambda^{2}, \quad k^{2} \equiv k_{\tilde{a}} k_{\tilde{a}} .
$$

Then the time derivative is replaced by

$$
\partial_{0} \equiv i \omega
$$

As a result, we arrive at the system of 4 linear homogeneous algebraic equations for $\Psi, \chi, Q, u$, which determines the dispersion relations. Equating the corresponding determinant to zero we get a fourth order equation for $\omega$ and then find the 4 dispersion relations. These are:

$$
\begin{aligned}
& \omega_{1,2}=\frac{i k^{2} \pm 2 \sqrt{-27 f^{4}-3 f^{2} k^{2}}}{6 f^{2}+k^{2}} \lambda, \\
& \omega_{3}=-\frac{2 i f^{2}}{\lambda}, \\
& \omega_{4}=\frac{2 i f^{2}\left(12 b f^{2}-2 b k^{2}+18 f^{2}-3 k^{2}\right)}{3 \lambda\left(8 b f^{2}+12 f^{2}-k^{2}\right)},
\end{aligned}
$$


where $b \equiv c_{3} / c_{5}$. We see that

$$
\begin{aligned}
\omega_{1,2} & \sim \lambda, \\
\omega_{3,4} & \sim \frac{f^{2}}{\lambda} .
\end{aligned}
$$

where $b \equiv c_{3} / c_{5}$. These "frequencies" are purely imaginary and naively there are instabilities. However, $\omega_{1}$ and $\omega_{2}$ correspond to the processes with characteristic time scale

$$
T \sim \frac{1}{\lambda}
$$

which means that the instability, if any, does not develop in the Hubble time (in fact, $\omega \sim \lambda$ contradicts our assumption (20), so the result (21) cannot be trusted). The other two "frequencies" are of order

$$
\omega_{3}, \omega_{4} \sim \frac{f^{2}}{\lambda} .
$$

In the limit of small $\lambda$ they are above a UV cutoff necessarily present in the model. Thus, within the region of validity of the effective low energy theory there are no propagating modes at all.

\section{Conclusion}

Interestingly, the spectrum of the theory about the self-accelerating background (at least its scalar part) is qualitatively different from the spectrum about the Minkowski background. This is not particularly surprising, since the two backgrounds are not connected by a family of solutions to the field equations, i.e., they belong to two separate branches. It is reassuring that the scalar perturbations about the selfaccelerating solution are healthy in the small- $\lambda$ limit.

We plan to consider tensor and vector sectors in future.

\section{Acknowledgements}

The author is indebted to V. Rubakov and S. Randjbar-Daemi for helpful discussions. This work has been supported by Russian Science Foundation grant 14-22-00161.

\section{References}

[1] T. Clifton, P. G. Ferreira, A. Padilla and C. Skordis, Phys. Rept. 513, 1 (2012) [arXiv:1106.2476 [astro-ph.CO]].

[2] G. R. Dvali, G. Gabadadze and M. Porrati, Phys. Lett. B 485, 208 (2000) [hep-th/0005016].

[3] N. Kaloper and D. Kiley, JHEP 0705, 045 (2007) [hep-th/0703190].

[4] T. Kobayashi, Phys. Rev. D 78, 084018 (2008) [arXiv:0806.0924 [hep-th]].

[5] C. de Rham, G. Dvali, S. Hofmann, J. Khoury, O. Pujolas, M. Redi and A. J. Tolley, Phys. Rev. Lett. 100, 251603 (2008) [arXiv:0711.2072 [hep-th]].

[6] N. Kaloper, Mod. Phys. Lett. A 23, 781 (2008) [arXiv:0711.3210 [hep-th]].

[7] G. Gabadadze, hep-ph/0308112.

[8] A. De Felice and S. Tsujikawa, Living Rev. Rel. 13, 3 (2010) [arXiv:1002.4928 [gr-qc]]. 
[9] Y. Fujii, K. Maeda, The Scalar-Tensor Theory of Gravitation (Cambridge University Press, 2003)

[10] S. Deser and A. Waldron, Phys. Rev. Lett. 87, 031601 (2001) [hep-th/0102166].

[11] S. Deser and A. Waldron, Phys. Lett. B 508, 347 (2001) [hep-th/0103255].

[12] M. Porrati, JHEP 0204, 058 (2002) [hep-th/0112166].

[13] C. de Rham, G. Gabadadze and A. J. Tolley, Phys. Rev. Lett. 106, 231101 (2011) [arXiv:1011.1232 [hep-th]].

[14] K. Hinterbichler, Rev. Mod. Phys. 84, 671 (2012) [arXiv:1105.3735 [hep-th]].

[15] V. A. Rubakov, hep-th/0407104.

[16] S. L. Dubovsky, JHEP 0410, 076 (2004) [hep-th/0409124].

[17] S. L. Dubovsky, P. G. Tinyakov and I. I. Tkachev, Phys. Rev. Lett. 94, 181102 (2005) [hepth/0411158].

[18] Z. Berezhiani, D. Comelli, F. Nesti and L. Pilo, Phys. Rev. Lett. 99, 131101 (2007) [hepth/0703264 [HEP-TH]].

[19] D. Blas, D. Comelli, F. Nesti and L. Pilo, Phys. Rev. D 80, 044025 (2009) [arXiv:0905.1699 [hep-th]].

[20] V. A. Rubakov and P. G. Tinyakov, Phys. Usp. 51, 759 (2008) [arXiv:0802.4379 [hep-th]].

[21] D. G. Boulware and S. Deser, Phys. Rev. D 6 (1972) 3368.

[22] C. Deffayet, Phys. Lett. B 502, 199 (2001) [hep-th/0010186].

[23] C. Deffayet, G. R. Dvali and G. Gabadadze, Phys. Rev. D 65, 044023 (2002) [astro-ph/0105068].

[24] M. A. Luty, M. Porrati and R. Rattazzi, JHEP 0309, 029 (2003) [hep-th/0303116].

[25] A. Nicolis and R. Rattazzi, JHEP 0406, 059 (2004) [hep-th/0404159].

[26] K. Koyama, Phys. Rev. D 72, 123511 (2005) [hep-th/0503191].

[27] D. Gorbunov, K. Koyama and S. Sibiryakov, Phys. Rev. D 73, 044016 (2006) [hep-th/0512097].

[28] C. Charmousis, R. Gregory, N. Kaloper and A. Padilla, JHEP 0610, 066 (2006) [hepth/0604086].

[29] K. Hayashi and T. Shirafuji, Prog. Theor. Phys. 64, 866 (1980) Erratum: [Prog. Theor. Phys. 65, 2079 (1981)].

[30] K. Hayashi and T. Shirafuji, Prog. Theor. Phys. 64, 1435 (1980) Erratum: [Prog. Theor. Phys. 66, 741 (1981)].

[31] K. Hayashi and T. Shirafuji, Prog. Theor. Phys. 64, 2222 (1980).

[32] E. Sezgin and P. van Nieuwenhuizen, Phys. Rev. D 21, 3269 (1980).

[33] V. P. Nair, S. Randjbar-Daemi and V. Rubakov, Phys. Rev. D 80, 104031 (2009) [arXiv:0811.3781 [hep-th]].

[34] V. Nikiforova, S. Randjbar-Daemi and V. Rubakov, Phys. Rev. D 80, 124050 (2009) [arXiv:0905.3732 [hep-th]].

[35] C. Deffayet and S. Randjbar-Daemi, Phys. Rev. D 84, 044053 (2011) [arXiv:1103.2671 [hepth]].

[36] V. Nikiforova, S. Randjbar-Daemi and V. Rubakov, arXiv:1606.02565 [hep-th]. 